\title{
New one-pot synthesis of 2-ylidenehydrazono-thiazoles
}

\author{
Aly, Ashraf A.
}

2019-11-02

Aly , A A , El-Sheref , E M , Brown , A B , Braese , S, Nieger , M \& Abdelhafez, E-S M N 2019 , ' New one-pot synthesis of 2-ylidenehydrazono-thiazoles ' , Journal of Sulfur Chemistry , vol. 40 , no. 6 , pp. 641-647 . https://doi.org/10.1080/17415993.2019.1635132

http://hdl.handle.net/10138/318178

https://doi.org/10.1080/17415993.2019.1635132

unspecified

acceptedVersion

Downloaded from Helda, University of Helsinki institutional repository.

This is an electronic reprint of the original article.

This reprint may differ from the original in pagination and typographic detail.

Please cite the original version. 


\section{New one-pot synthesis of 2-ylidenehydrazono-thiazoles}

\section{Ashraf A. Aly, ${ }^{* a}$ Essmat M. El-Sheref, ${ }^{\text {a,b }}$ Alan B. Brown, ${ }^{c}$ Stefan Bräse $^{\mathrm{d}}$, Martin Nieger ${ }^{\mathrm{e}}$ and El-Shimaa M. N. Abdelhafez ${ }^{\mathrm{f}}$}

${ }^{a}$ Chemistry Department, Faculty of Science, Minia University, 61519 El-Minia, Egypt. ${ }^{b}$ Chemistry Department, College of Sciences and Humanities, Sattam bin Abdulaziz University, P.O. Box 83, Al-Kharij 11942, Saudi Arabia. ${ }^{C}$ Chemistry Department, Florida Institute of Technology, 150 W University Blvd, Melbourne, Florida 32901, USA. ${ }^{d}$ Institute of Organic Chemistry, Karlsruhe Institute of Technology, FritzHaber-Weg 6, 76131 Karlsruhe, Germany. ${ }^{e}$ Department of Chemistry, University of Helsinki, PO Box 55

(A.I. Virtasen aukio 1), FIN-00014 Helsinki, Finland. ${ }^{f}$ Medicinal Chemistry Department, Faculty of Pharmacy, Minia University, 61519 El-Minia, Egypt

Abstract. A new one pot reaction of substituted thiosemicarbazides with 2-bromoacetophenone and carbonyl compounds gave 2-hydrazonothiazoles in good yields. The structures of the isolated compounds were corroborated by NMR, IR, mass spectra and elemental analyses in addition to X-ray structure

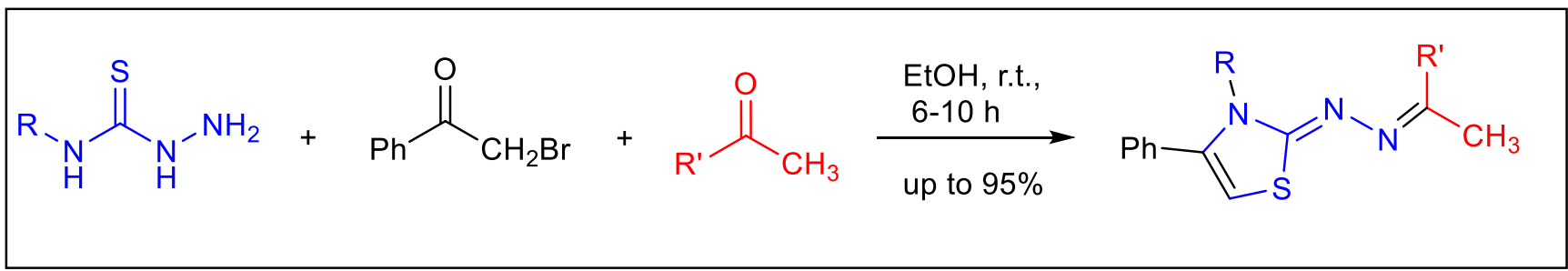

Keywords: Substituted thiosemicarbazides, 2-bromoacetophenone, carbonyl compounds, 2-ylidinehydrazinothiazoles, NMR, X-ray.

\section{Introduction}

1,3-thiazole moiety is one of the most important scaffolds in heterocyclic chemistry and drug design and discovery [1]. It is widely found in diverse pharmacologically active substances and in some naturallyoccurring compounds [1]. It was reported that substituted 2-hydrazino-1,3-thiazoles show an inhibitory activity (MIC of $161 \mu \mathrm{g} / \mathrm{mL}$ ) against a resistant strain of Candida krusei [2]. Few other 2-hydrazino-1,3thiazole analogues that exhibit significant antimicrobial activity have been recently reported in the literature [3]. Thiazoles bearing a bicyclic or heterocyclic ring on the hydrazone function and a phenyl at C-4 position of the thiazole nucleus are particularly active and present micromolar or submicromolar MIC against several 
Candida spp. Strains (repeated) [1]. Thus, such scaffolds could be seen as a starting point for further optimization of novel antifungal agents.

Thiazoles and their analogues serve also as precursors for the synthesis of biologically active compounds [4]. They were reported to possess antimicrobial [5-8], analgesic [9], anti-inflammatory [10], anticonvulsant [11], cardiotonic [12], anticancer [13-15], antitubercular [16] and anthelmintic [17] effects. (it is better to start with this paragraph) Several methods for the synthesis of thiazole derivatives have been developed [1820], the most widely used is being the Hantzsch synthesis, which uses thioamides and $\alpha$-halocarbonyl (or $\alpha$ halo ester) compounds as starting materials [21].

It was previously shown that ylidenes of hydrazinothiazoles could be synthesized by two different methods:

a) reaction of hydrazinothiazoles with the appropriate ketones or aldehydes or b) reaction between thiosemicarbazides and 2-bromoacetophenones. The yields of the methods described have been suffered from long time of reaction. Our recent publication includes the synthesis of versatile, hitherto thiazole derivatives resembling LY293111 [22]. Besides that, we reported on thiazole synthesis via donor-acceptor reactions [23-25]. To address these interesting biological and pharmaceutical properties and their synthetic utility, we aim to design a new facile method in order to prepare ylidenes of hydrazonothiazoles in a one pot reaction starting from substituted thiosemicarbazides, 2-bromoacetophenones and ketones.

\section{Results and Discussion}

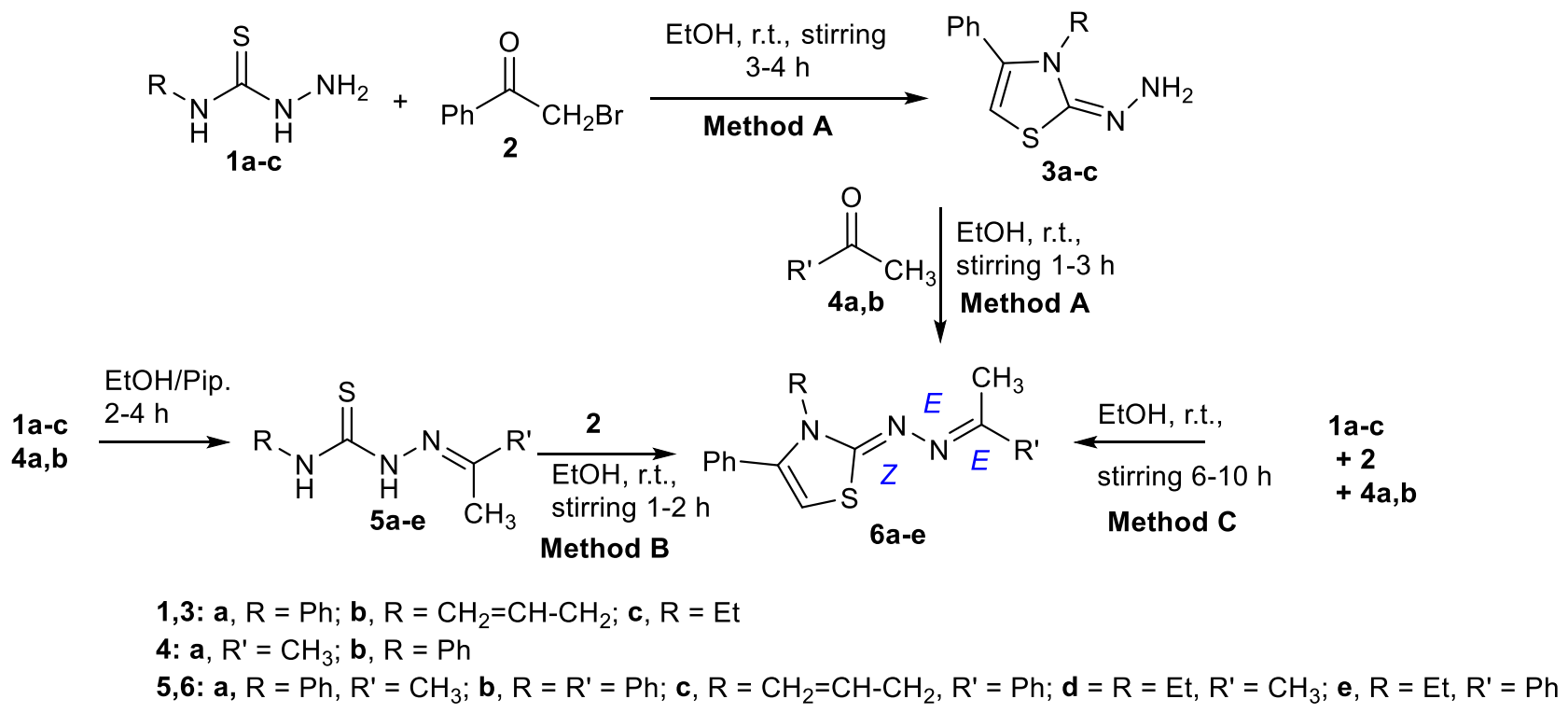

Scheme 1. Synthesis of 2-ylidenes of hydrazonothiazoles 6a-e 
The compounds 3a-c [26] were previously prepared on one hand by reaction of substituted thiosemicarbazides 1a-c with 2-bromo-acetophenone (2) (Scheme 1, method A). On the other hand, reaction of 1a-c with appropriate ketones $\mathbf{4 a , b}$, gave the corresponding thiosemihydrazones 5a-e [27] and upon subjecting compounds 5a-e with bromide 2, the corresponding 2-ylidenes of hydrazinothiazoles 6a-e were obtained in moderate yields (Scheme 1, method B). The yields of the products 6a-e (i.e. preparation of compounds 6a-d according to lit [28]) using the two aforesaid procedures were not optimal (Scheme 1).

We herein reported on one-pot method using a mixture of 1a-c, 2 and $\mathbf{4 a}, \mathbf{b}$ (Scheme 1, method C). The yields of compounds 6a-e using the method $\mathbf{C}$ are shown in Table 1. The distinctive carbons of the aforesaid compounds are shown in Figure 1. The structures of the isolated products were confirmed by mass, NMR, IR spectra in addition to elemental analyses. For example, the NMR spectroscopic data of compounds $6 \mathbf{a}$ and $\mathbf{6 b}$ are shown in Tables 2 and 3.

Table 1. Yields of compounds 6a-e using method $\mathbf{C}$

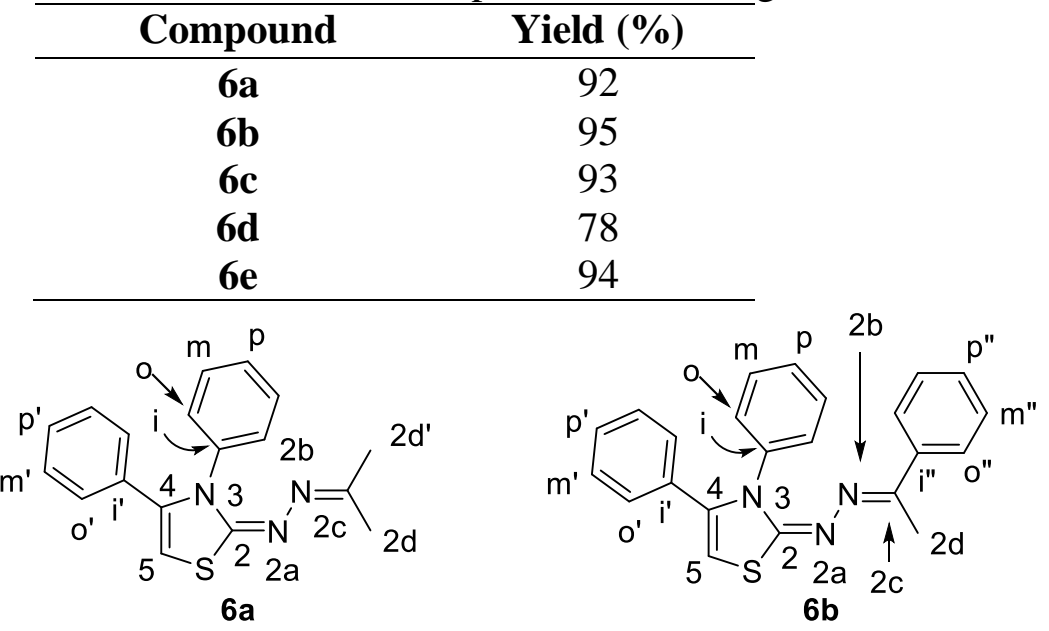

Figure 1. Distinctive carbons of compounds $\mathbf{6 a}$ and $\mathbf{6 b}$

Table 2. NMR spectroscopy of $6 a$

\begin{tabular}{|c|c|c|}
\hline${ }^{1}$ H NMR: & COSY: & Assignment \\
\hline $7.33($ ("t", $J=7.5 ; 2 \mathrm{H})$ & $7.22,7.15$ & $\mathrm{H}-m$ \\
\hline $7.22(\mathrm{~m} ; 6 \mathrm{H})$ & $7.33,7.15$ & $\mathrm{H}-o, m^{\prime}, p, p^{\prime}$ \\
\hline $7.15(\mathrm{~m} ; 2 \mathrm{H})$ & $7.33,7.22$ & $\mathrm{H}-o^{\prime}$ \\
\hline $6.51(\mathrm{~s} ; 1 \mathrm{H})$ & & $\mathrm{H}-5$ \\
\hline $1.91(\mathrm{~s} ; 3 \mathrm{H})$ & & $\mathrm{H}-2 \mathrm{~d} / 2 \mathrm{~d}^{\prime}$ \\
\hline $1.79(\mathrm{~s} ; 3 \mathrm{H})$ & & $H-2 d^{\prime} / 2 d$ \\
\hline${ }^{13}$ C NMR: & HMBC: & Assignment: \\
\hline 165.94 & $6.51,1.91,1.79$ & $\mathrm{C}-2$ \\
\hline 158.86 & $1.91,1.79$ & $\mathrm{C}-2 \mathrm{c}$ \\
\hline $139.08,138.06$ & $7.33,7.22,7.15,6.51$ & $\mathrm{C}-i, 4$ \\
\hline
\end{tabular}




\begin{tabular}{|c|c|c|c|}
\hline 131.05 & & $7.22,7.15,6.51$ & $\mathrm{C}-i^{\prime}$ \\
\hline 128.52 & 7.33 & $7.33,7.22,7.15,6.51$ & $\mathrm{C}-m$ \\
\hline $128.32,128.19$ & 7.22 & $7.33,7.22,7.15,6.51$ & $\mathrm{C}-o, m^{\prime}$ \\
\hline 128.14 & 7.22 & $7.33,7.22,7.15,6.51$ & C- $p / p^{\prime}$ \\
\hline 127.89 & 7.15 & $7.33,7.22,7.15,6.51$ & $\mathrm{C}-O$ \\
\hline 127.23 & 7.22 & $7.33,7.22,7.15,6.51$ & $\mathrm{C}-p^{\prime} / p$ \\
\hline 100.95 & 6.51 & & $\mathrm{C}-5$ \\
\hline 24.30 & 1.91 & 1.79 & $C-2 d / 2 d^{\prime}$ \\
\hline 18.17 & 1.79 & 1.91 & $\mathrm{C}-2 \mathrm{~d}^{\prime} / 2 \mathrm{~d}$ \\
\hline${ }^{15}$ N NMR: & HSQC: & HMBC: & Assignment: \\
\hline 344.5 & & $1.91,1.79$ & $\mathrm{~N}-2 \mathrm{~b}$ \\
\hline 138.5 & & $7.22,6.51$ & N-3 \\
\hline
\end{tabular}

Table 3. NMR spectroscopy of compound $6 \mathrm{~b}$

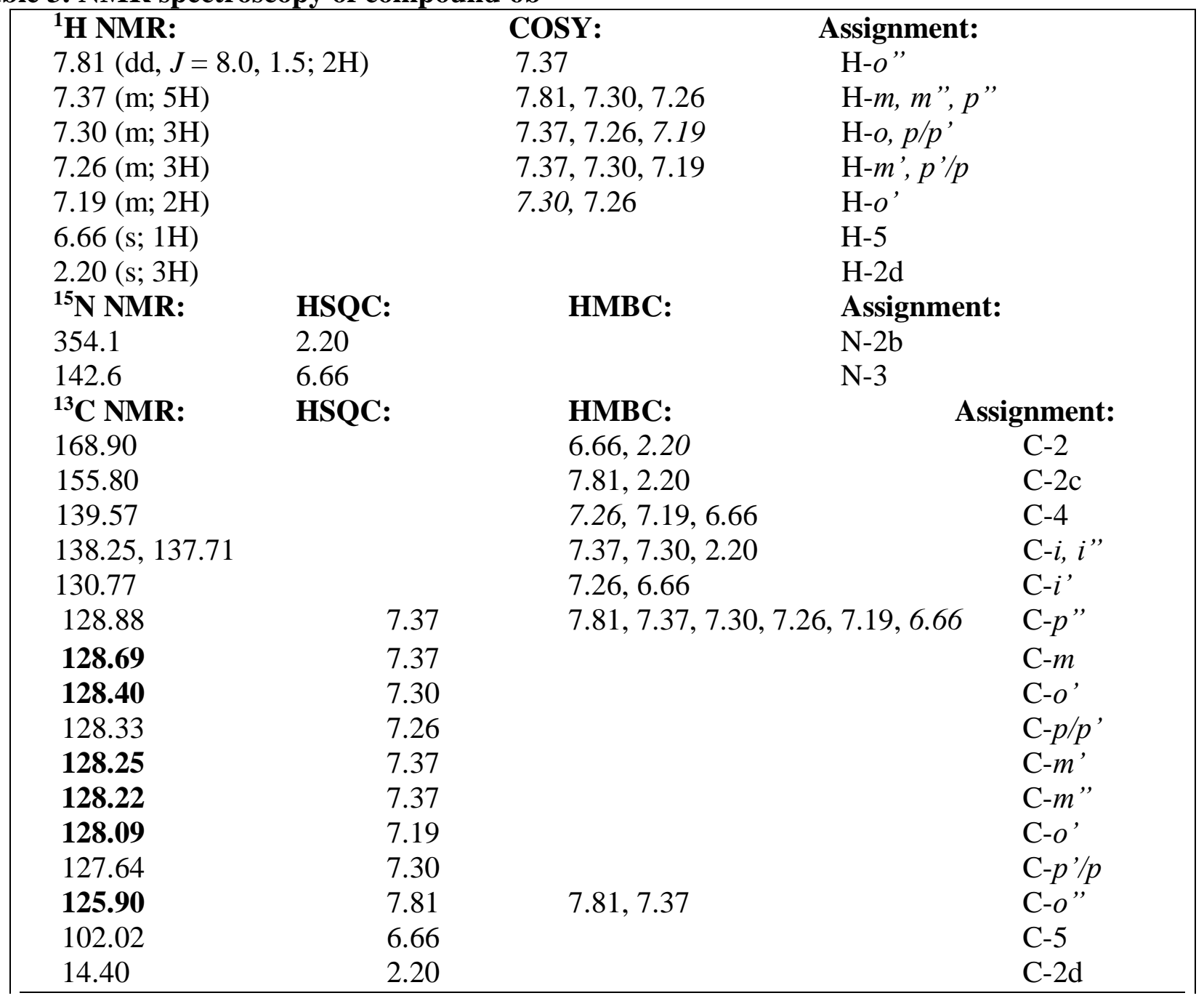

The X-ray structure analysis confirmed the structure of $\mathbf{6 a}$ as shown in Figure 2. The stereochemistry of compound 6a was determined with a Z, s-trans (transoid) configuration for the CNN moiety and the 
compound was identified finally as (Z,s-trans)-3,4-diphenyl-propan-2-ylidenehydrazono-2,3-dihydrothiazole.

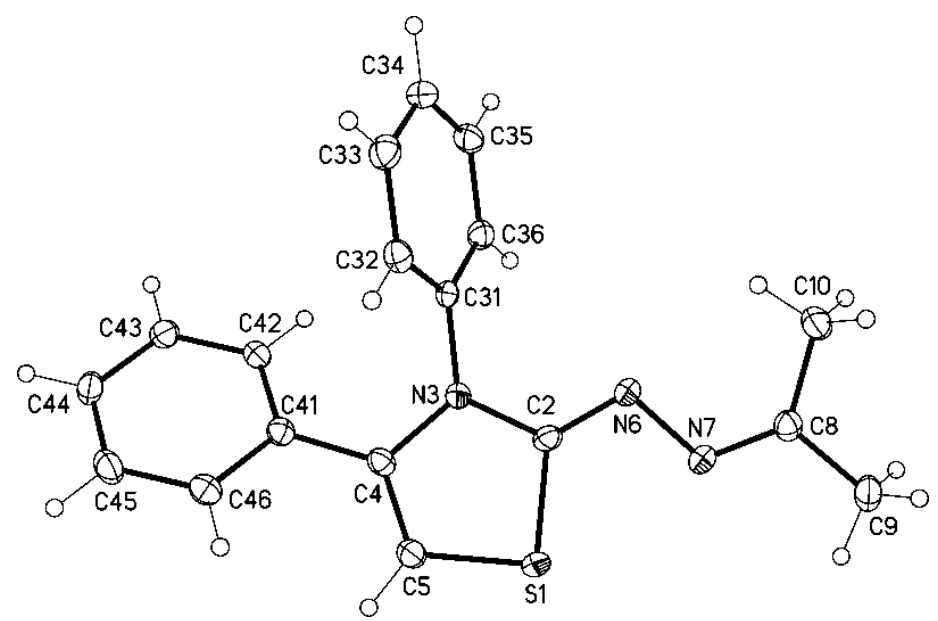

Figure 1. Molecular structure of $\mathbf{6 a}$ (displacement parameters are drawn at 50\% probability level)

\section{Experimental Part}

\subsection{General}

Melting points were determined using an APP Digital ST 15 melting point apparatus and uncorrected. TLC analyses were performed using analytical Merck 9385 silica aluminum sheets (Kieselgel 60) with $\mathrm{PF}_{254}$ indicator. The IR spectra were recorded as KBr disks on Shimadzu-408 infrared spectrophotometer, Faculty of Science, Minia University. The NMR spectra were measured using a Bruker AV-400 spectrometer at the Karlsruher Institut für Technologie (KIT), Institute of Organic Chemistry, Karlsruhe, Germany. Chemical shifts were expressed as $\delta$ (ppm) with tetramethylsilane as internal reference. The samples were dissolved in DMSO- $d_{6}, \mathrm{~s}=$ singlet, $\mathrm{d}=$ doublet, $\mathrm{dd}=$ doublet of doublet and $\mathrm{t}=$ triplet. Mass spectrometry were recorded on a Varian MAT 312 instrument in EI mode $(70 \mathrm{eV})$, at the Karlsruhe Institut für Technologie (KIT), Institute of Organic Chemistry, Karlsruhe, Germany. Elemental analyses were carried out using Varian Elementary device in National Research Center, Giza, Egypt.

\subsection{Crystal Structure Determination of 6a}

The single-crystal X-ray diffraction study was carried out on a Bruker D8 Venture diffractometer with

Photon100 detector at $123(2) \mathrm{K}$ using $\mathrm{Cu}-\mathrm{K} \alpha$ radiation $(\lambda=1.54178 \AA$ ). Dual space methods (SHELXT) [29] were used for structure solution and refinement was carried out using SHELXL-2014 (full-matrix least- 
squares on $F^{2}$ ) [29]. Hydrogen atoms were refined using a riding model. A semi-empirical absorption correction and an extinction correction were applied.

6a: Yellow crystals, $\mathrm{C}_{18} \mathrm{H}_{17} \mathrm{~N}_{3} \mathrm{~S}, M_{\mathrm{r}}=307.40$, crystal size $0.20 \times 0.08 \times 0.04 \mathrm{~mm}$, monoclinic, space group $P 2{ }_{1} / c$ (No. 14), $a=5.8645(2) \AA, b=14.0488(5) \AA, c=18.7533(7) \AA, \beta=97.003(2)^{\circ}, V=1533.11(10) \AA^{3}, Z$ $=4, \rho=1.332 \mathrm{Mg} / \mathrm{m}^{-3}, \mu\left(\mathrm{Cu}-\mathrm{K}_{\alpha}\right)=1.857 \mathrm{~mm}^{-1}, F(000)=648,2 \theta_{\max }=144.4^{\circ}, 16566$ reflections, of which 3015 were independent $\left(R_{\text {int }}=0.025\right), 202$ parameters, $R_{1}=0.029$ (for $\left.2856 \mathrm{I}>2 \sigma(\mathrm{I})\right)$, w $R_{2}=0.072$ (all data), $S=1.04$, largest diff. peak / hole $=0.255 /-0.240$ e $\AA^{-3}$.

CCDC 1896104 (6a) contains the supplementary crystallographic data for this paper. These data can be obtained free of charge from The Cambridge Crystallographic Data Centre via www.ccdc.cam.ac.uk/data request/cif.

\subsection{Starting materials}

Compounds 3a-c and 5a-e were prepared according to literature [26] and [27], respectively (see their corresponding references). Compounds 6a-d were prepared for comparison according to literature [28].

\subsection{General procedure describes preparation of compounds 6a-e}

A mixture of 1a-c, 2 and $\mathbf{4 a}, \mathbf{b}(1 \mathrm{mmol})$ in absolute ethanol (EtOH, $150 \mathrm{~mL})$, was stirred at room temperature for 6-10 h (the reaction was monitored by TLC). The reaction mixture was allowed to stand overnight. The formed products were recrystallized from the stated solvents.

(Z)-3,4-Diphenyl-2-(propan-2-ylidenehydrazono)-2,3-dihydrothiazole (6a). Pale yellow crystals (EtOH), $\mathrm{R}_{\mathrm{f}}=0.5$ (Toluene: Ethyl acetate: 10:1), yield $0.283 \mathrm{~g}(92 \%)$, m.p. $165-167{ }^{\circ} \mathrm{C}$ (m.p. [28a] $165{ }^{\circ} \mathrm{C}$ ). NMR $\left(\mathrm{CDCl}_{3}\right)$ : see Table 2. Anal. Calcd. For $\mathrm{C}_{18} \mathrm{H}_{17} \mathrm{~N}_{3} \mathrm{~S}$ (307.42): C, 70.33; H, 5.57; N, 13.67. Found: C, 70.22; $\mathrm{H}, 5.45 ; \mathrm{N}, 13.55$.

(Z)-3,4-Diphenyl-2-((E)-1-phenylethylidene)hydrazono)-2,3-dihydrothiazole (6b). Pale yellow crystals $(\mathrm{EtOH}), \mathrm{R}_{\mathrm{f}}=0.4$ (Toluene: Ethyl acetate: 10:1), yield $0.350 \mathrm{~g}(95 \%)$, m.p. $310-312^{\circ} \mathrm{C}$ (m.p. [28b] 310-312 $\left.{ }^{\circ} \mathrm{C}\right)$. NMR $\left(\mathrm{CDCl}_{3}\right)$ : see Table 3. Anal. calcd. for $\mathrm{C}_{23} \mathrm{H}_{19} \mathrm{~N}_{3} \mathrm{~S}$ (369.49): C, 74.77; H, 5.18; N, 11.37. Found: C, 74.62; H, 5.00; N, 11.25. 
(Z)-3-Allyl-4-phenyl-2-(E)-1-phenylethylidene)hydrazono)-2,3-dihydrothiazole (6c). Pale yellow crystals

$\left(\mathrm{CH}_{3} \mathrm{CN}\right), \mathrm{R}_{\mathrm{f}}=0.7$ (Toluene: Ethyl acetate: 10:1), yield $0.310 \mathrm{~g}(93 \%)$, m.p. 247-249 ${ }^{\circ} \mathrm{C}$ (m.p. [28b] 246$248{ }^{\circ} \mathrm{C}$ ). Anal. calcd. for $\mathrm{C}_{20} \mathrm{H}_{19} \mathrm{~N}_{3} \mathrm{~S}$ (333.45): C, 72.04; H, 5.74; N, 12.60. Found: C, 72.16; H, 5.80; N, 12.46 .

(Z)-3-Ethyl-4-phenyl-2-propan-2-ylidene)hydrazono)-2,3-dihydrothiazole (6d). Pale yellow crystals $\left(\mathrm{CH}_{3} \mathrm{OH}\right), \mathrm{R}_{\mathrm{f}}=0.75$ (Toluene: Ethyl acetate: 10:1), yield $0.202 \mathrm{~g}(78 \%)$, m.p. $=97-98{ }^{\circ} \mathrm{C}\left(\right.$ lit $\left.[28 \mathrm{~b}] 96{ }^{\circ} \mathrm{C}\right)$. Anal. calcd. for $\mathrm{C}_{14} \mathrm{H}_{17} \mathrm{~N}_{3} \mathrm{~S}$ (259.37): C, 64.83; H, 6.61; N, 16.20. Found: C, 64.70; H, 6.81; N, 16.36 .

(Z)-3-Ethyl-4-phenyl-2-(E)-1-phenylethylidene)hydrazono)-2,3-dihydrothiazole (6e). Pale yellow crystals $\left(\mathrm{CH}_{3} \mathrm{OH}\right), \mathrm{R}_{\mathrm{f}}=0.8$ (Toluene: Ethyl acetate: 10:1), yield $0.302 \mathrm{~g}(94 \%)$, m.p. $=212-214{ }^{\circ} \mathrm{C} .{ }^{1} \mathrm{H}$ NMR (400 MHz, CDCl 3$): \delta=7.80-7.78(\mathrm{~m}, 2 \mathrm{H}, \mathrm{Ph}-\mathrm{H}), 7.60-7.40(\mathrm{~m}, 4 \mathrm{H}, \mathrm{Ph}-\mathrm{H}), 6.92(\mathrm{~s}, 1 \mathrm{H}$, thiazole-5), 6.86-6.82 (m, 4H, Ph-H), 4.00 (q, 2H, J=7.0 Hz, $\mathrm{CH}_{2}$-ethyl), 2.40 (s, 3H, $\left.\mathrm{CH}_{3}\right), 1.20(\mathrm{t}, 3 \mathrm{H}, J=7.0 \mathrm{~Hz}$, $\mathrm{CH}_{3}$-ethyl). ${ }^{13} \mathrm{C}$ NMR (400 MHz, $\left.\mathrm{CDCl}_{3}\right): \delta=164.0,155.0(\mathrm{C}=\mathrm{N}), 139.0$ (C-4-thiazole), $137.0(\mathrm{Ph}-2 \mathrm{C})$, 132.0, 131.0, 130.6, 130.0 (Ph-2CH), 127.0, 126.2 (Ph-CH), 102.0 (Thiazole-CH-5), 38.9 (CH${ }_{2}$-ethyl), 21.0 $\left(\mathrm{CH}_{3}\right)$, $13.0\left(\mathrm{CH}_{3}\right)$. Anal. calcd. for $\mathrm{C}_{19} \mathrm{H}_{19} \mathrm{~N}_{3} \mathrm{~S}$ (321.44): C, 71,00; H, 5.96; N, 13.07. Found: C, 71.10; $\mathrm{H}$, $6.00 ; \mathrm{N}, 13.12$.

\section{Conclusion}

In this paper, we illustrate a new method of synthesizing hydrazonothiazoles by one-pot reaction of substituted thiosemicarbazides, 2-bromoacetophenones and ketones. The advantages of our new method are; a) high yield percentages of the products, b) facile one pot method (at room temperature) and c) the illustrated method does not involve any additives.

\section{Acknowledgements}

The NMR spectrometer at Florida Institute of Technology was purchased with assistance from the U.S. National Science Foundation (CHE 03-42251).

\section{Disclosure statement}

No potential conflict of interest was reported by the authors. 


\section{References}

[1] Chimenti F, Bizzarri B, Bolasco, A, Secci D, Chimenti P, Granese A, Carradori S, D’Ascenzio AM, Lilli D, Rivanera D. Synthesis and biological evaluation of novel 2,4-disubstituted-1,3-thiazoles as antiCandida spp. Agents. Eur. J. Med. Chem. 2011;46:378-382.

[2] Chimenti F, Bizzarri B, Maccioni E, Secci D, Bolasco A, Fioravanti A, Chimenti P, Granese, A, Carradori S, Rivanera D, Lilli D, Zicari A, Distinto S. Synthesis and in vitro activity of 2thiazolylhydrazone derivatives compared with the activity of clotrimazole against clinical isolates of Candida spp. Bioorg. Med. Chem. Lett. 2007;17:4635-4650.

[3] Secci D, Bizzarri B, Bolasco A, Carradori S, D’Ascenzio M, Rivanera D.; Mari E, Polletta L, Zicari A. Synthesis, anti-Candida activity, and cytotoxicity of new (4-(4-iodophenyl)thiazol-2-yl)hydrazine derivatives. Eur. J. Med. Chem. 2012;53:246-253.

[4] De Logu, A.; Saddi, M.; Cardia, M. C.; Borgna, R.; Sanna, C.; Saddi, E. Maccioni, J. Antimicrob. Chemother. 2005, 55, 692.

[5] Ali, I.; Lone, M. N.; Al-Othman, Z. A.; Al-Warthan, A.; Sanagi. M. M. Curr. Drug Target 2015, 16, 711.

[6] Tiperciuc, B.; Zaharia, V.; Colosi, I.; Moldovan, C.; Crisan, O.; Pirnau, A.; Vlase, L.; Duma, M.; Oniga. O. J. Heterocyl. Chem. 2011, 49, 1407.

[7] Ali, E.-S. T.; El. Kazak, A. M. Eur. J. Chem. 2010, 1, 6-11.

[8] Pawar CD, Sarkate AP, Karnik KS, Bahekar SS, Pansare DN, Shelke RN, Jawale CS, Shinde DB. Bioorg. Med. Chem. Lett. 2016, 26, 3525-3528

[9] Mohammad, H.; Reddy, P. V. N.; Monteleone, D.; Mayhoub, A. S.; Cushman, M.; Seleem, M. N. Eur. J. Med. Chem. 2015, 94, 306-316

[10] Thore, S. N.; Gupta, S. V.; Baheti, K. G. J. Saudi Chem. Soc. 2016, 20, 46-S52.

[11] Nastasă, C.; Tiperciuc, B.; Pârvu, A.; Duma, M.; Ionuţ, I.; Oniga, O. Arch. Pharm. Chem. Life Sci. 2013, $346,481-490$.

[12] Das, D.; Sikdar, P.; Bairagi, M. Eur. J. Med. Chem. 2016, 109, 89-98 
[13] Duan, L. M.; Yu, H. Y.; Jia, C. J.; Li. Y. L. Bioorg. Med. Chem. 2015, 23, 6111-6117

[14] Turan-Zitouni, G.; Altıntop, M. D.; Ozdemir, A.; Kaplancıklı, Z. A.; Çiftçi, G. A.; Temel. H. E. Eur. J. Med. Chem. 2016, 107, 288-294

[15] Parekh, N. M.; Mistry, B. M.; Pandurangan, M.; Shinde, S. K.; Patel, R. V. Chin. Chem. Lett. 2017, 28, $602-606$

[16] Xie, X. X.; Li, H.; Wang, J.; Mao, S.; Xin, M. H.; Lu, S. M.; Mei, Q. B.; Zhang, S. Q. Bioorg. Med. Chem. 2015, 23, 6477-6485.

[17] Dhumal, S. T.; Deshmukh, A. R.; Bhosle, M. R.; Khedkar,V. M.; Nawale, L. U.; Sarkar, D.; Mane, R. A. Bioorg. Med. Chem. Lett. 2016, 26, 3646-3651

18] Amnerkar, N. D.; Bhongade, B. A.; Bhusari, K. P. Arab. J. Chem. 2015, 8, 545-552.

[19] Karabasanagouda, T.; Adhikari, A. V.; Ramgopal, D.; Parameshwarappa, G. Ind. J. Chem. 2008, 47, $144-152$

[20] Amir, A.; Kumar, H.; Khan, S. A. Bioorg. Med. Chem. Lett. 2008, 18, 918-922

[21] Nassar Sci. Synthesis, (in vitro) Antitumor and Antimicrobial Activity of some Pyrazoline, Pyridine, and Pyrimidine Derivatives Linked to Indole Moiety. E. J. Am. 2010;6:463-471

[22] Aly AA, Ibrahim MAA, El-Sheref EM, Hassan AMA, Brown AB. Prospective new amidinothiazoles as leukotriene B4 inhibitors. J. Mol. Structure 2019; 1175: 414-427.

[23] Aly, A. A.; Hassan, A. A.; Bräse, S.; Ibrahim, M. A. A.; Al-Latif, El-S. S. M.; Spuling, E.; Nieger, M. J. Sulfur Chem. 2017, 38, 69-75

[24] Aly AA, Ishak EA, Brown AB. J. Sulfur Chem. 2014:35, 382-393

[25] Aly, A. A.; Brown, A. B.; Abdel-Aziz, M.; Abuo-Rahma, G. El-D.A. A.; Radwan, M. F.; Ramadan, M.; Gamal-Eldeen, A. M. J. Heterocycl. Chem. 2010, 47, 547-554

[26] (a) 3a: Hünig, S.; Lampe, W. J. Prakt. Chem. 1959, 8, 264-278. (b) 3b,c: Pasquier, C.; Duc-Reichlin, N.; Braun, H.-J. (to Proctor \& Gamble and Wella A-G) PCT Int. Appl. 2005, WO 2006094217 A2 (Chem. Abstr. 145, 320760). 
[27] (a) 5a: Stephen, H.W.; Wilson, F.J. J. Chem. Soc. 1926, 2531-2538. (b) 5b: Stephen, H. W.; Wilson, F. J. J. Chem. Soc. 1928, 1415-1422. (c) 5c: Iradyan, M.A.; Aroyan, R.A.; Avakimyan, D.A.; Stepanyan, G.M. Pharm. Chem. J. 2011, 45, 26-29. (e) 5d: Walter, W.; Rohloff, C. Justus Liebigs Ann. Chem. 1977, 447-462. (f) 5e: El-Shazly, R.M.; Al-Hazmi, G.A.A.; Ghazy, S.E.; El-Shahawi, M.S.; El-Asmy, A.A. Spectrochim. Acta, A 2005, 61, 243-252.

[28] (a) 6a: Pfeiffer, W.-D.; Junghans, D.; Saghyan, A.S.; Langer, P. J. Heterocycl. Chem. 2014, 51, $1063-$ 1067. (b) 6b: Hassan, A.A.; Ibrahim, Y.R.; El-Sheref, E. M.; Abdel-Aziz, M.; Bräse, S.; Nieger, M. Arch. Pharm. (Weinheim) 2013, 346, 562-570. (c) 6d: Reference 28b. (d) 6e: Pfeiffer, W.-D.; Ahlers, K.-D.; Saghyan, A.S.; Villinger, A.; Langer, P. Helv. Chim. Acta 2014, 97, 76-87.

[29] Sheldrick GM. SHELXT - Integrated space-group and crystal-structure determination. Acta Crystallogr. 2015; A71:3-8. 